Elect. Comm. in Probab. 10 (2005), 60-69

\title{
POISSON THINNING BY MONOTONE FACTORS
}

\author{
KAREN BALL
}

Department of Mathematics, Indiana University, Rawles Hall, Bloomington, IN 47405

email: kareball@indiana.edu

Submitted June 18, 2004, accepted in final form March 11, 2005

AMS 2000 Subject classification: 60G55, 37A35

Keywords: Poisson processes, stochastic domination, factor maps

\section{Abstract}

Let $X$ and $Y$ be Poisson point processes on $\mathbb{R}$ with rates $\lambda_{1}, \lambda_{2}$ respectively. We show that if $\lambda_{1}>\lambda_{2}$, then there exists a deterministic map $\phi$ with $\phi(X) \stackrel{d}{=} Y$ such that the joint distribution of $(X, \phi(X))$ is translation-invariant and which is monotone in the sense that for all intervals $I, \phi(X)(I) \leq X(I)$, almost surely.

\section{Introduction}

Let $X$ be a Poisson point processes on $\mathbb{R}$ with rate $\lambda_{1}$. If $\lambda_{2}<\lambda_{1}$, then it is well-known that a Poisson point process $Y$ with rate $\lambda_{2}$ can be obtained from $X$ by thinning; points are independently deleted from $X$ with probability $1-\lambda_{2} / \lambda_{1}$ to get $Y$. This gives a coupling of the two processes $X$ and $Y$, which is monotone in the sense that almost surely all of the points in $Y$ are also points in $X$. Another way of saying this is that for any interval $I, X(I) \geq Y(I)$ almost surely. Note also that the joint distribution of $(X, Y)$ under the thinning construction is translation-invariant. In ergodic theory, a shift-invariant coupling of two processes is called a joining.

In this paper, we show that the set of translation-invariant monotone couplings of Poisson processes $X$ and $Y$ with rates $\lambda_{1}>\lambda_{2}$ includes the special class of translation-invariant couplings which are supported on graphs of functions. In other words, we prove that there is a deterministic map $\phi$ such that

1. $\phi(X) \stackrel{d}{=} Y$,

2. the joint distribution of $(X, \phi(X))$ is invariant under translations, and

3. $X(I) \geq \phi(X)(I)$ for all intervals $I$, almost surely.

Such a map $\phi$ is called a monotone factor from $X$ to $Y$. A factor is a map satisfying just the first two conditions.

Our map $\phi$ will have the additional property of being finitary, which means that it is almost surely continuous in the vague topology. A finitary map $\phi$ is nice because $\left.\phi(X)\right|_{I}$ can be 
estimated accurately with finite data, for example by computer. We also show, in Section 6 , that for any $I$ the expected size of the interval $J(X)$ needed to compute $\left.\phi(X)\right|_{I}$ is finite. The main theorem of this paper is the following:

Theorem 1.1. Let $X$ and $Y$ be Poisson point processes on $\mathbb{R}$ with rates $\lambda_{1}$ and $\lambda_{2}$ respectively. If $\lambda_{1}>\lambda_{2}$, then there is a monotone finitary factor $\phi$ taking $X$ to $Y$.

There is a discrete-time version of the question of the existence of a monotone factor from $X$ to $Y$. A discrete-time version of Theorem 1.1 is proved in [1], though more conditions are needed for this case. The construction of the monotone factor in discrete-time is more complicated than the continuous-time construction given here. This is mostly because there is more randomness in finite intervals of Poisson processes than there is in finite intervals of Bernoulli processes.

The discrete-time problem was posed to the author by Russ Lyons. The question in the context of Poisson point processes was asked by Alexander Holroyd and Yuval Peres. These problems are interesting in part because they combine stochastic domination (see Strassen [7]) and coupling ideas from probability theory with a question about the existence of factors, which have long been of interest in ergodic theory.

One nice property of the monotone factor we construct here is that it is explicit - given $\lambda_{1}, \lambda_{2}$, and a third number $T$, which can be chosen as a function of $\lambda_{1}$ and $\lambda_{2}$, one can compute $\phi(X)$. The author knows of no other explicit factor taking one Poisson point process to another. The obvious map $X(t) \mapsto X\left(\lambda_{2} t / \lambda_{1}\right)$ is not a factor because its joint distribution is not invariant under translations.

Many ideas in this paper are related to the constructions of Keane and Smorodinski in [4] and [5]. In these papers, they prove that if $\bar{X}$ and $\bar{Y}$ are i.i.d. processes with finite alphabets and if $\bar{X}$ has more entropy than $\bar{Y}$ then there is a finitary factor from $\bar{X}$ to $\bar{Y}$ and furthermore, if $\bar{X}$ and $\bar{Y}$ have the same entropy, then the finitary factor can be taken to be invertible. We are able to use the properties of Poisson processes to simplify things, and so the construction we end up with is significantly different from theirs. Other studies which have been made of factors of Poisson processes and more general point processes in $\mathbb{R}^{d}$ include [2], [3], and [8], in which the factors of interest were factor graphs, which are translation- or isometry-equivariant functions of the process which place a graph structure on the points of the process.

The structure of the paper is as follows. In Section 2, we give formal definitions some of the concepts in this introduction and set up notation that will be used throughout the paper. In Sections 3-5, we construct $\phi$ and prove that it is a finitary monotone factor. We start by constructing a monotone coupling $\gamma$ of the processes $X$ and $Y$ restricted to a finite interval. This coupling $\gamma$ has the property that when $X$ has exactly one point in the interval, it can only couple to $Y$ with zero points in the interval. We then construct a monotone coupling $\rho$ of $X$ and $Y$ on all of $\mathbb{R}$ which projects to $\gamma$ on certain intervals. Thus, for $\rho$-almost every pair $(X, Y)$, we will be able to pick out certain points from $X$ which are not in $Y$. Randomness from these deleted points is then used to decide which other points from $X$ should or should not be included in $\phi(X)$. In this way, we are able to construct our factor $\phi$. In Section 6 , we show that $\left.\phi(X)\right|_{I}$ depends on $X_{J}$ for some interval $J$ with finite expected length. We conclude in Section 7 with a couple of open questions related to this work.

\section{Notation and definitions}

We start by formalizing the definitions of couplings, monotonicity, and factors. 
Definition 2.1. A coupling of two measures $\mu$ and $\nu$ on $\Omega_{1}$ and $\Omega_{2}$, respectively, is a measure $\gamma$ on $\Omega_{1} \times \Omega_{2}$ such that $\mu(\cdot)=\gamma\left(\cdot \times \Omega_{2}\right)$ and $\nu(\cdot)=\gamma\left(\Omega_{1} \times \cdot\right)$.

A coupling of two random processes $X$ and $Y$ is a joint distribution for $(X, Y)$ which is a coupling of the distributions of $X$ and $Y$.

If $\mu$ and $\nu$ are measures on a common space $\Omega$ and if $\leq i s$ a partial order on $\Omega$, then a coupling $\gamma$ of $\mu$ and $\nu$ is monotone if

$$
\gamma\left(\left\{\left(\omega_{1}, \omega_{2}\right): \omega_{1} \geq \omega_{2}\right\}\right)=1 .
$$

Let $\mathcal{B}(\mathbb{R})$ be the Borel $\sigma$-algebra on $\mathbb{R}$ and let $\Omega$ be the space of Borel measures on $\mathbb{R}$. Define a partial order $\leq$ on $\Omega$ by

$$
\omega_{1} \leq \omega_{2} \Longleftrightarrow \int F d \omega_{1} \leq \int F d \omega_{2} \quad \forall \text { continuous functions } F: \mathbb{R} \rightarrow \mathbb{R} .
$$

If $X$ and $Y$ are point processes, which we may regard as processes taking values in $\Omega$, a coupling of $X$ and $Y$ is monotone with respect to this partial order if and only if $X(I) \geq Y(I)$ for all intervals $I$ almost surely (or equivalently if all of the points in $Y$ are also points in $X$, almost surely).

Factors are maps which behave well with respect to certain dynamics. In our case, the dynamics we are concerned with are translations on $\mathbb{R}$. Let $\left(\sigma_{t}\right)_{t \in \mathbb{R}}$ be the group of translations or leftshifts on $\mathbb{R}$, with $\sigma_{t}(r)=r-t$. Then $\sigma_{t}$ acts on $\Omega$ by $\sigma_{t}(\omega)(A)=\omega\left(\sigma_{t}^{-1}(A)\right)=\omega(A+t)$ where $A \in \mathcal{B}(\mathbb{R})$.

Definition 2.2. Let $X$ and $Y$ be stationary processes taking values in $\Omega$. $A$ factor from $X$ to $Y$ is a map $\phi: \Omega \rightarrow \Omega$ (defined a.s. with respect to the distribution of $X$ ), such that

$$
\begin{aligned}
\phi(X) & \stackrel{d}{=} Y \text { and } \\
\sigma_{t}(\phi(X)) & =\phi\left(\sigma_{t}(X)\right) \forall t \in \mathbb{R}, \text { a.s. }
\end{aligned}
$$

$A$ factor $\phi$ from $X$ to $Y$ is monotone if $\mathbf{P}(X \geq \phi(X))=1$.

A little thought shows that (2) is equivalent to requiring that the joint distribution of $(X, \phi(X))$ be invariant under translations by $t$ for all $t \in \mathbb{R}$.

The third important definition we need is that of a finitary factor.

Definition 2.3. Let $X$ and $Y$ be stationary processes taking values in $\Omega$. A factor $\phi$ from $X$ to $Y$ is finitary if there is a set $\tilde{\Omega} \subseteq \Omega$ with $\mathbf{P}_{X}(\tilde{\Omega})=1$ such that $\left.\phi\right|_{\tilde{\Omega}}$ is continuous in the vague topology on $\Omega$ (see, for example, Reiss' book [6] for the definition of vague convergence).

From now on, $X$ is a Poisson point process on $\mathbb{R}$ with rate $\lambda_{1}$ which we regard as a random measure taking values in $\Omega$. Let the support of the random measure $X$ be the random set

$$
[X]=\{x \in \mathbb{R}: X(x)=1\} .
$$

We will also use the notation $[\omega]$ to denote the support of a nonrandom measure $\omega \in \Omega$. Let $\left(\chi_{n}\right)_{n \in \mathbb{Z}}$ be a sequence of random variables giving the ordered locations of the points in $[X]$. Let $\mu$ be the distribution of $X$ on $\Omega$. Define $Y,[Y],\left(\psi_{n}\right)$, and $\nu$ to be an Poisson process with rate $\lambda_{2}$, its support, the locations of its points, and its distribution. Assume that $\lambda_{1}>\lambda_{2}$.

Poisson processes are supported on counting measures, where the atoms are discrete and each atom has mass 1. Call the set of such measures $\Omega^{\prime} \subset \Omega$. Then $\mu\left(\Omega^{\prime}\right)=\nu\left(\Omega^{\prime}\right)=1$. For $A \in \mathcal{B}(\mathbb{R})$, define $\Omega_{A}=\{\omega \in \Omega: \omega(A)=\omega(\mathbb{R})\}$ and $\Omega_{A}^{\prime}=\Omega^{\prime} \cap \Omega_{A}$. If $\omega \in \Omega$, we put $\omega_{A}(\cdot)=\omega(\cdot \cap A)$. 


\section{Monotone couplings of Poisson processes}

As mentioned in the introduction, a natural monotone coupling of $X$ and $Y$ is given by thinning $X$ to get $Y$ as follows. Let $\left(\alpha_{n}\right)_{n \in \mathbb{Z}}$ be a sequence of i.i.d. random variables independent of $X$ taking value 1 with probability $\lambda_{2} / \lambda_{1}$ and 0 otherwise. Use the points $\left(\chi_{n}\right)$ of $X$ and $\left(\alpha_{n}\right)$ to define a random measure $Y$ supported on $\Omega^{\prime}$ by

$$
Y=\sum_{n \in \mathbb{Z}} \alpha_{n} \mathbf{1}_{\chi_{n}}
$$

In other words, form the process $Y$ by taking $[X]$ and independently deleting points with probability $1-\lambda_{2} / \lambda_{1}$. It is standard and easy to prove that the point process $Y$ defined in this way is Poisson with rate $\lambda_{2}$. Call the resulting monotone coupling of $X$ and $Y \pi$ and let $\pi_{t}$ be the restriction of $\pi$ to an interval of length $t$.

The first step in the proof of Theorem 1.1 is to give another monotone coupling of $X$ and $Y$ with some special properties. Actually, this $\gamma$ will only couple $X$ and $Y$ restricted to an interval in $\mathbb{R}$ with finite length $T$. Fix $T>0$, so that

$$
e^{\left(\lambda_{1}-\lambda_{2}\right) T}>1+\lambda_{1} T
$$

or equivalently, keeping in mind that $X$ and $Y$ are Poisson processes,

$$
\left.\mathbf{P}\left(\#\left[Y_{(0, T]}\right]=0\right)>\mathbf{P} \#\left[X_{(0, T]}\right] \leq 1\right)
$$

Let $\mu_{T}$ and $\nu_{T}$ be the distributions of $X_{(0, T]}$ and $Y_{(0, T]}$, respectively. The coupling $\gamma$ of $\mu_{T}$ and $\nu_{T}$ is then constructed as follows. Let

$$
\begin{aligned}
r_{i} & =\mathbf{P}\left(\#\left[X_{(0, T]}\right]<i\right) \text { for } i=0,1,2, \ldots \\
s_{i} & =\mathbf{P}\left(\#\left[Y_{(0, T]}\right]<i\right) \text { for } i=0,1,2, \ldots
\end{aligned}
$$

Since $\lambda_{1}>\lambda_{2}$, we have $r_{i} \leq s_{i}$ for all $i$. By inequality (4), $r_{2}<s_{1}$. Define a probability measure $\gamma^{*}$ on $\mathbb{N}_{0} \times \mathbb{N}_{0}$ by

$$
\gamma^{*}(i, j)=\ell\left(\left[r_{i}, r_{i+1}\right] \cap\left[s_{j}, s_{j+1}\right]\right)
$$

where $\ell$ is Lebesgue measure. Then $\gamma^{*}(\{(i, j): i \geq j\}=1$.

Define a probability measure supported on $\Omega_{(0, T]}^{\prime} \times \Omega_{(0, T]}^{\prime}$ by taking $\omega_{1}$ with distribution $\mu_{T}$ and

$$
\begin{aligned}
& \gamma\left(\left(\omega_{1}, \omega_{2}\right) \mid \omega_{1} \times \Omega\right)= \\
& \begin{cases}\gamma^{*}\left(\left(\#\left[\omega_{1}\right], \#\left[\omega_{2}\right]\right) \mid \#\left[\omega_{1}\right] \times \mathbb{N}\right) \cdot\left(\begin{array}{l}
\#\left[\omega_{1}\right] \\
\#\left[\omega_{2}\right]
\end{array}\right)^{-1} & \omega_{2} \leq \omega_{1}, \omega_{2} \in \Omega_{(0, T]}^{\prime} \\
0 & \text { otherwise. }\end{cases}
\end{aligned}
$$

In other words, the first marginal of $\gamma$ has distribution $\mu_{T}$ and for a given $\omega_{1}$, the number of points in the support of $\omega_{2}$ is chosen from $\gamma^{*}$ conditioned on the number of points in the support of $\omega_{1}$. Then a subset of $\left[\omega_{1}\right]$ of the appropriate size is chosen uniformly at random to get $\left[\omega_{2}\right]$.

Lemma 3.1. The measure $\gamma$ defined above is a monotone coupling of $\mu_{T}$ and $\nu_{T}$. 
Proof. It is clear from the definition that $\gamma$ is a monotone coupling of its first and second marginals and that it must have $\mu_{T}$ as its first marginal as desired. Thus, we just have to show that the second marginal is $\nu_{T}$. By the way in which we constructed $\gamma^{*}$,

$$
\gamma\left(\left\{\Omega \times\left\{\omega_{2}\right\}: \#\left[\omega_{2}\right]=i\right\}\right)=e^{-T \lambda_{2}}\left(T \lambda_{2}\right)^{i} / i !=\mathbf{P}\left(\#\left[Y_{(0, T]}\right]=i\right)
$$

so the number of points in $\gamma(\Omega \times \cdot)$ has the right distribution. Furthermore, since $X$ is a Poisson process, once $\#\left[X_{(0, T]}\right]$ is fixed, the points in $[X] \cap(0, T]$ are distributed uniformly and independently in the interval $(0, T]$. If we chose a random subset of these points, they will also be distributed uniformly and independently in $(0, T]$. This proves that the second marginal of $\gamma$ is indeed the Poisson point process on $(0, T]$ with rate $\lambda_{2}$.

Remark 3.2. The important feature of $\gamma$ that we will use in this paper is that $\gamma^{*}(1,0)=$ $\mathbf{P}\left(\#\left[X_{(0, T]}\right]=1\right)$ (this follows from the fact that $s_{1}>r_{2}$, as noted above). This means that $\mu_{T}$-almost any $\omega_{1}$ with $\#\left[\omega_{1}\right]=1$ couples under $\gamma$ only to the zero measure $\varphi$ with $\varphi(\mathbb{R})=0$. Thus, if there is exactly point in $\left[\omega_{1}\right]$, it will never be included in $\left[\omega_{2}\right]$ under $\gamma$. The fact that we know this point will be deleted will give us the information we need to construct a monotone factor out of the monotone coupling we will construct.

\section{Markers and more monotone couplings}

We now construct a new monotone coupling $\rho$ of $X$ and $Y$ using both the natural monotone coupling $\pi$ of $\mu$ and $\nu$ and the specially constructed monotone coupling $\gamma$ of $\mu_{T}$ and $\nu_{T}$. In the following section, we will then show how to use $\rho$ to construct a monotone finitary factor from $X$ to $Y$.

First, we introduce the notion of a marker. For $\omega \in \Omega$, let $\bar{a}(\omega)=\{a:[\omega] \cap(a-2 T, a]=\{a\}\}$. Then $\mu_{t}$-almost surely there are infinitely many points in $\bar{a}(\omega)$. We will inductively form a subset of $\bar{a}(\omega)$ as follows. Let

$$
\begin{aligned}
& a^{(0)}(\omega)=\{a \in \bar{a}(\omega): \bar{a}(\omega) \cap(a-3 T, a)=\emptyset\}, \\
& a^{(n)}(\omega)=\left\{a: \exists b \in a^{(n-1)}(\omega) \text { s.t. } a=\min \left\{a^{\prime} \in \bar{a}(\omega): a^{\prime} \geq b+3 T\right\}\right\} .
\end{aligned}
$$

Set $a(\omega)=\cup_{n=0}^{\infty} a^{(n)}(\omega)$. We call the points in $a(\omega)$ markers. Markers are important because they will indicate a change in $\rho$ from coupling according to $\pi$ to coupling according to $\gamma$. The rather complicated definition of $a(\omega)$ ensures that the distance between markers is always at least $3 T$ and that if $a_{i}$ is a marker, the next marker $a_{i+1}$ is the smallest element of $\bar{a}(\omega)$ which is larger than $a_{i}+3 T$.

Let $Z=\left(Z^{(1)}, Z^{(2)}\right)$ and $\tilde{Z}=\left(\tilde{Z}^{(1)}, \tilde{Z}^{(2)}\right)$ be random pairs of measures taking values in $\Omega \times \Omega$, with distribution $\gamma$ and $\pi$ respectively, and suppose that $Z$ and $\tilde{Z}$ are independent. Define a new random pair of measures $\bar{Z}=\left(\bar{Z}^{(1)}, \bar{Z}^{(2)}\right)$ by taking

$$
\begin{aligned}
& \bar{Z}^{(1)}:=Z_{(0, T]}^{(1)}+\tilde{Z}_{(T, \infty)}^{(1)} \\
& \bar{Z}^{(2)}:=Z_{(0, T]}^{(2)}+\tilde{Z}_{(T, \infty)}^{(2)}
\end{aligned}
$$

$\bar{Z}$ is a coupling of $X_{\mathbb{R}^{+}}$and $Y_{\mathbb{R}^{+}}$since both $Z$ and $\tilde{Z}$ couple $X$ and $Y$ restricted to the appropriate intervals and since $Z$ and $\tilde{Z}$ are independent and Poisson processes have independent 
increments. Let $\tau$ be the random stopping time which is the first time such that $\tau$ is the endpoint of a marker in $\bar{Z}^{(1)}$; i.e.

$$
\tau:=\min \left\{t>3 T:\left[\bar{Z}^{(1)}\right] \cap(t-2 T, t]=\{t\}\right\} .
$$

Let $W=\left(W^{(1)}, W^{(2)}\right)$ be the random pair of measures on $\mathbb{R}$ obtained by setting

$$
W^{(1)}:=\bar{Z}_{(0, \tau]}^{(1)}, \quad W^{(2)}:=\bar{Z}_{(0, \tau]}^{(2)} .
$$

With respect to $W, \tau=\max \left\{t: t \in\left[W^{(1)}\right]\right\}$.

Let $W_{0}, W_{1}, \ldots$ be i.i.d. with $W_{i} \stackrel{d}{=} W$ for all $i$. Each $W_{i}$ then defines random time $\tau_{i}$ which is the largest $t$ in the support of $W_{i}^{(1)}$. Define $\widehat{Z}=\left(\widehat{Z}^{(1)}, \widehat{Z}^{(2)}\right)$ by

$$
\widehat{Z}:=W_{0}+\sum_{i=1}^{\infty} \sigma_{-\left(\sum_{j=0}^{i-1} \tau_{j}\right)}\left(W_{i}\right) .
$$

Since Poisson processes have independent increments, $\widehat{Z}$ is also a monotone coupling of $X_{\mathbb{R}^{+}}$ and $Y_{\mathbb{R}^{+}}$. Let $\hat{\rho}$ be the distribution of $\widehat{Z}$. This measure $\hat{\rho}$ is not stationary because we started by coupling from $\gamma$, so let $\dot{\rho}=\lim _{n \rightarrow \infty} \frac{1}{n} \sum_{j=0}^{n-1} \sigma_{j}(\hat{\rho})$. Then $\dot{\rho}$ is a stationary coupling of $X$ and $Y$ restricted to $\mathbb{R}^{+}$. This implies that $\dot{\rho}$ is a measure on $\Omega_{\mathbb{R}^{+}} \times \Omega_{\mathbb{R}^{+}}$which is invariant with respect to the semigroup action of $\left(\sigma_{t}\right)_{t \in \mathbb{R}^{+}}$on this space:

$$
\sigma_{t}\left(\omega, \omega^{\prime}\right)=\left(\left(\sigma_{t}(\omega)\right)_{\mathbb{R}^{+}},\left(\sigma_{t}\left(\omega^{\prime}\right)\right)_{\mathbb{R}^{+}}\right) .
$$

Therefore, there exists a translation-invariant measure $\rho$ on $\Omega \times \Omega$ such that the map $\left(\omega, \omega^{\prime}\right) \mapsto$ $\left(\omega_{\mathbb{R}^{+}}, \omega_{\mathbb{R}^{+}}^{\prime}\right)$ takes $\rho$ to $\dot{\rho}$. This measure $\rho$ is the natural extension of $\dot{\rho}$. This implies that the following lemma holds.

Lemma 4.1. The probability measure $\rho$ is a monotone translation-invariant coupling of $X$ and $Y$.

The construction of $\rho$ given above is designed to make Lemma 4.1 obvious. We now give another useful characterization of $\rho$. First, we need some notation. Let $a(\omega)=\left(a_{i}\right)_{i \in \mathbb{Z}}$ be the sequence of consecutive markers in $X$. Let $J_{i}=\left(a_{i}, a_{i}+T\right]$ for each $i$. Since markers at least distance $3 T$ apart, these intervals are all disjoint. Let $K_{i}=\left(a_{i}+T, a_{i+1}\right]$; these are the intervals interlaced between the $J_{i}$. Finally, $k_{i}$ is the length of the interval $K_{i}$, which must be at least $2 T$. It is important to keep in mind that the sequences $\left(a_{i}\right),\left(J_{i}\right),\left(K_{i}\right)$, and $\left(k_{i}\right)$ are all deterministic functions of the random process $X$ and that the sequences (though not the indexing) are equivariant under translations; for example $a\left(\sigma_{t}(X)\right)=\sigma_{t}(a(X))$.

We want to show that $\rho(\omega \times \cdot \mid \omega \times \Omega)$ has a certain product structure. Take sets $A_{i} \in \sigma\left(\Omega_{J_{i}}\right)$ and $B_{i} \in \sigma\left(\Omega_{K_{i}}\right)$ and set

$$
C_{\mathbf{A}, \mathbf{B}}=\bigcap_{i \in \mathbb{Z}}\left\{\omega \in \Omega: \omega_{J_{i}} \in A_{i}, \omega_{K_{i}} \in B_{i}\right\} .
$$

For any such set, it is clear from the construction of $\rho$ that

$$
\begin{aligned}
\rho\left(\{\omega\} \times C_{\mathbf{A}, \mathbf{B}} \mid \omega \times \Omega\right)= & \prod_{i=-\infty}^{\infty} \gamma\left(\sigma_{-a_{i-1}}\left(\left\{\omega_{J_{i}}\right\} \times A_{i}\right) \mid \omega_{J_{i}}\right) \\
& \cdot \pi_{k_{i}}\left(\sigma_{-\left(a_{i-1}+T\right)}\left(\left\{\omega_{K_{i}}\right\} \times B_{i}\right) \mid \omega_{K_{i}}\right) .
\end{aligned}
$$

In other words, to sample from $\rho$ given first coordinate $\omega$, we select the second coordinate independently from $\gamma$ given $\omega_{J_{i}}$ on each $J_{i}$ and from $\pi_{k_{i}}$ given $\omega_{K_{i}}$ on each $K_{i}$. 


\section{Construction of a monotone factor}

In this section, we use the joining $\rho$ to construct a monotone factor $\phi$ taking $X$ to $Y$.

For $\mu_{T}$-almost every $\omega \in \Omega, \gamma\left(\omega \times \omega^{\prime} \mid \omega\right)$ is positive for finitely many $\omega^{\prime}$, all of which have $\left[\omega^{\prime}\right] \subseteq[\omega]$. Similarly, for $\mu_{t}$-almost every $\omega, \pi_{t}\left(\omega \times \omega^{\prime} \mid \omega\right)$ is positive for the finitely many $\omega^{\prime}$ with $\left[\omega^{\prime}\right] \subseteq[\omega]$. We define $\phi$ so that it chooses among these finitely many possibilities on each $J_{i}$ and $K_{i}$. The next lemma constructs the functions that make these choices.

Lemma 5.1. If $U$ is a uniform random variable, there are measurable functions $f: \Omega^{\prime} \times$ $[0,1] \rightarrow \Omega^{\prime}$ and $\left(g_{t}: \Omega^{\prime} \times[0,1] \rightarrow \Omega^{\prime}\right)_{t>0}$ such that

$$
\begin{array}{rr}
f(\omega, U) \text { has distribution } \gamma((\omega, \cdot) \mid \omega \times \Omega), & \mu_{T} \text {-a.s. } \\
g_{t}(\omega, U) \text { has distribution } \pi_{t}((\omega, \cdot) \mid \omega \times \Omega), & \mu_{t}-a . s .
\end{array}
$$

Proof. Define the lexicographic ordering on the set $\left\{\omega \in \Omega^{\prime}:[\omega]<\infty\right\}$ :

$$
\begin{aligned}
\omega<\omega^{\prime} & \text { if } \#[\omega]<\#\left[\omega^{\prime}\right] \text { or } \\
\text { if } \#[\omega] & =\#\left[\omega^{\prime}\right], \omega \neq \omega^{\prime} \text { and } \min \left\{t: t \in[\omega] \triangle\left[\omega^{\prime}\right]\right\} \in[\omega],
\end{aligned}
$$

where $\Delta$ denotes the symmetric difference. This is a total ordering on this set.

To define $f$ and $g$, let $\omega_{1}<\cdots<\omega_{m}$ be the measures in $\Omega$ with $\left[\omega_{i}\right] \subseteq[\omega]$. Then set

$$
\begin{gathered}
f(\omega, r)=\omega_{i} \text { if } \gamma\left(\{\omega\} \times\left\{\omega_{j}: j<i\right\} \mid \omega\right) \leq r<\gamma\left(\{\omega\} \times\left\{\omega_{j}: j \leq i\right\} \mid \omega\right), \\
g_{t}(\omega, r)=\omega_{i} \text { if } \pi_{t}\left(\{\omega\} \times\left\{\omega_{j}: j<i\right\} \mid \omega\right) \leq r<\pi_{t}\left(\{\omega\} \times\left\{\omega_{j}: j \leq i\right\} \mid \omega\right) .
\end{gathered}
$$

These functions are clearly measurable and satisfy (6) and (7).

If $\omega \in \Omega_{(0, T]}^{\prime}$ and $\#[\omega]=1$ then by Remark 3.2, $\gamma(\{\omega\} \times \cdot \mid \omega \times \Omega)$ is concentrated on the point $(\omega, \varphi)$, where $\varphi$ is the measure of total mass 0 . Therefore for such $\omega, f(\omega, U) \equiv \varphi$ is independent of $U$. It is also independent of the locations of the point in [ $\omega]$. This is the key fact that we will use in constructing our monotone factor map $\phi$ from $X$ to $Y$.

We have defined $a(\omega)=\left\{a_{i}\right\}$ to be the set of markers for $\omega$. Let $i(\omega)=\left\{i_{n}\right\}$ be the set of integers $i_{n}$ with $\#\left[\omega_{J_{i_{n}}}\right]=1$. Both sets are defined $\mu$-almost surely. Set

$$
A(\omega)=\left\{\omega^{\prime}: a\left(\omega^{\prime}\right)=a(\omega), i\left(\omega^{\prime}\right)=i(\omega), \omega_{\mathbb{R} \backslash\left(\cup_{n} J_{i_{n}}\right)}^{\prime}=\omega_{\mathbb{R} \backslash\left(\cup_{n} J_{i_{n}}\right)}\right\} .
$$

This defines the $\sigma$-algebra which identifies measures which agree everywhere on $\mathbb{R}$ except on the intervals $J_{i_{n}}$. For $\omega^{\prime} \in A(\omega)$, if $i_{n} \in i(\omega)$, set $\left[\omega_{J_{i_{n}}}^{\prime}\right]=\left\{\bar{\chi}_{n}\right\}$. We get a sequence of random variables as a function of $X$, conditional on $A(\omega)$, by taking

$$
U_{n}:=\left(\bar{\chi}_{n}-a_{i_{n}}\right) / T
$$

Lemma 5.2. $\left(U_{n}\right)_{n \in \mathbb{Z}}$ is an i.i.d. sequence of uniform random variables.

Proof. Because $X$ is a Poisson point process, conditional on the location of $a_{i_{n}}, \bar{\chi}_{n}$ is independent of $X_{\mathbb{R} \backslash\left[a_{i_{n}}, a_{i_{n}}+T\right)}$. The only thing we know about $\bar{\chi}_{n}$ is that it is the unique point in $[X]$ in the interval $\left(a_{i_{n}}, a_{i_{n}}+T\right)$. It is a standard result that for a Poisson process, such a point is uniformly distributed in $\left(a_{i_{n}}, a_{i_{n}}+T\right)$. Therefore, $U_{n}$ is uniformly distributed in $(0,1)$. The independence of increments in a Poisson process implies that the $U_{n}$ are independent. 
Definition of $\phi$ : We are now ready to define our monotone factor $\phi$. Since each $U_{n}$ can be written as a sequence of i.i.d. Bernoulli- $(1 / 2,1 / 2)$ random variables, we can redistribute the bits of this expansion to get a sequence of i.i.d. uniform random variables $U_{n}^{(1)}, U_{n}^{(2)}, \ldots$. Define

$$
\phi(\omega)_{J_{i}}= \begin{cases}\sigma_{-a_{i}} f\left(\sigma_{a_{i}}\left(\omega_{J_{i}}\right), U_{m}^{(2 j)}\right) \text { for } i_{m}<\left(i=i_{m}+j\right)<i_{m+1} & i \notin(i(\omega)) \\ \varphi & i \in(i(\omega)) .\end{cases}
$$

and

$$
\phi(\omega)_{K_{i}}=\sigma_{-\left(a_{i}+T\right)} g_{k_{i}}\left(\sigma_{a_{i}+T}\left(\omega_{K_{i}}\right), U_{m}^{(2 j+1)}\right) \text { for } i_{m} \leq\left(i=i_{m}+j\right)<i_{m+1} .
$$

This defines $\phi(\omega)$ on all of $\mathbb{R}$ almost surely.

To complete the proof of the main theorem, we must show that this map $\phi$ is a monotone finitary factor from $X$ to $Y$.

Proof of Main Theorem 1.1. Clearly, $\phi$ is a monotone, measurable map, defined $\mu$-almost surely on $\Omega$, and it commutes with shifts $\sigma_{t}$ for all $t$.

It is also not difficult to see that $\phi$ is finitary. This follows from the fact that for each $n$, $\phi(\omega)_{\left(a_{i_{n}}, a_{i_{n-1}}\right)}$ depends only and continuously on $\omega_{\left(a_{i_{n}}, a_{i_{n-1}}\right)}$.

It only remains to show that $\phi \mu=\nu$. Let $\mu_{\omega}(\cdot)=\mu(\cdot \mid A(\omega))$. Define $\nu_{\omega}(\cdot)=\rho(\Omega \times \cdot \mid$ $A(\omega) \times \Omega)$. Then it follows from the fact that $\rho$ is a coupling of $\mu$ and $\nu$ that $\int \nu_{\omega} d \mu(\omega)=\nu$. Thus it suffices to show that that for $\mu$-almost every $\omega$,

$$
\left.\phi\right|_{A(\omega)} \mu_{\omega}=\nu_{\omega} .
$$

By $(5), \nu_{\omega}$ is a product measure:

$$
\nu_{\omega}(\cdot)=\prod_{i \in \mathbb{Z}} \gamma\left(\Omega \times \cdot \mid\left\{\omega_{J_{i}}\right\} \times \Omega\right) \times \pi_{k_{i}}\left(\Omega \times \cdot \mid\left\{\omega_{K_{i}}\right\} \times \Omega\right) .
$$

We chose $f$ and $g_{t}$ so that $\left(\phi \mu_{\omega}\right)_{J_{i}}=\left(\nu_{\omega}\right)_{J_{i}}$ and $\left(\phi \mu_{\omega}\right)_{K_{i}}=\left(\nu_{\omega}\right)_{K_{i}}$ for all $i \in \mathbb{Z}$. The $\left(U_{n}^{(j)}\right)_{n, j}$ are i.i.d. and uniform by Lemma 5.2. The independence of the $U_{n}^{(j)}$ implies that $\phi \mu_{\sigma}$ has the desired product structure as in (10). Thus, $\phi \mu_{\omega}=\nu_{\omega}$ and $\phi$ is a monotone factor from $X$ to $Y$.

\section{How finitary is $\phi$ ?}

Here we compute an estimate for the expected distance between $a_{i_{n}}$ and $a_{i_{n+1}}$. This distance is important because $\phi(X)_{\left(a_{n}, a_{i_{n}+1}\right]}$ is a function of $X_{\left(a_{i_{n}}, a_{i_{n+1}}\right]}$. In particular, we show that the size of this interval has finite expected value.

\section{Proposition 6.1.}

$$
\mathbf{E}\left(a_{i_{n+1}}-a_{i_{n}}\right)<e^{T \lambda_{1}}\left(T \lambda_{1}\right)^{-1}\left(e^{2 T \lambda_{1}} / \lambda_{1}+3 T\right)<\infty .
$$


Proof. We first estimate $\mathbf{E}\left(a_{i+1}-a_{i}\right)$. If $Z_{1}, Z_{2}, \ldots$ are i.i.d. exponential random variables with mean $1 / \lambda_{1}$ and $\tau$ is the first $t$ such that $Z_{t} \geq 2 T$, then

$$
\begin{aligned}
\mathbf{E}\left(a_{i+1}-a_{i}\right) & =T+\mathbf{E}\left(\sum_{t=1}^{\tau} Z_{t}\right) \\
& =T+\mathbf{E}(\tau-1) \mathbf{E}\left(Z_{1} \mid Z_{1}<2 T\right)+\mathbf{E}\left(Z_{\tau} \mid Z_{\tau} \geq 2 T\right) \\
& <\mathbf{E}(\tau) \mathbf{E}\left(Z_{1}\right)+3 T=e^{2 T \lambda_{1}} / \lambda_{1}+3 T
\end{aligned}
$$

The last equality above follows from the fact that $\tau$ is a geometric random variable with mean $\left(\mathbf{P}\left(Z_{1} \geq 2 T\right)\right)^{-1}=e^{2 T \lambda_{1}}$.

The probability that $\#\left[\omega_{J_{i}}\right]=1$ is $e^{-T \lambda_{1}}\left(T \lambda_{1}\right)$. Therefore,

$$
\mathbf{E}\left(a_{i_{n+1}}-a_{i_{n}}\right) \leq e^{T \lambda_{1}}\left(T \lambda_{1}\right)^{-1}\left(e^{2 T \lambda_{1}} / \lambda_{1}+3 T\right) .
$$

Remark 6.2. Recall that $T$ was chosen in (3) so that

$$
e^{\left(\lambda_{1}-\lambda_{2}\right) T}>1+\lambda_{1} T \text {. }
$$

For fixed $\lambda_{1}$, as $\lambda_{2} \nearrow \lambda_{1}, T$ must tend to infinity and the expected size of the interval blows up. On the other hand, when $\lambda_{2} \ll \lambda_{1}$ a reasonable choice for $T$ may be made. For instance, for $\lambda_{1}=1$ and $\lambda_{2}<1-\ln (2) \approx .3068$, we may take $T=1$ and get the estimate $\mathbf{E}\left(a_{i_{n+1}}-a_{i_{n}}\right)<e\left(e^{2}+3\right)<29$. This shows that, at least when $\lambda_{2}$ is small, the numbers involved here are not astronomical.

\section{$7 \quad$ Some open problems}

We conclude with a couple of open problems relating to this work.

The joint distribution of $(X, \phi(X))$ is not invariant under the map $\eta: \mathbb{R} \rightarrow \mathbb{R}$ given by $\eta(a)=-a$ and so is not a factor with respect to the full group of isometries of $\mathbb{R}$.

Question 7.1. Does there exist a monotone map $\phi$ from $X$ to $Y$ such that the joint distribution of $(X, \phi(X))$ is invariant with respect to the group of isometries of $\mathbb{R}$ ?

It is also natural to ask the analogous question in higher dimensions.

Question 7.2. If $X$ and $Y$ are Poisson point processes in $\mathbb{R}^{d}$ for $d \geq 2$ with intensities $\lambda_{1}>\lambda_{2}$, does there exist a monotone factor from $X$ to $Y$ with respect to either the group of translations on $\mathbb{R}^{d}$ or the group of isometries on $\mathbb{R}^{d}$ ?

Acknowledgements. Thank you to Russ Lyons, Alexander Holroyd, and Yuval Peres for asking interesting questions. We also thank the referee for his valuable comments.

\section{References}

[1] Ball, K. Monotone factors of i.i.d. processes, to appear in the Israel J. Math.

[2] Ferrari, P.A., Landim, C., Thorisson, H. Poisson trees, succession lines and coalescing random walks, Ann. I. H. Poincaré-PR 40 (2004), 141-152. 
[3] Holroyd, A., Peres, Y. Trees and matchings from point processes, Elect. Comm. in Probab. 8 (2003), 17-27.

[4] Keane, M., Smorodinsky, M. A class of finitary codes, Israel J. Math. 26 (1977) nos. 3-4, 352-371.

[5] Keane, M., Smorodinsky, M. Bernoulli schemes of the same entropy are finitarily isomorphic, Ann. Math. 109 (1979), 397-406.

[6] Reiss, R.-D. A course on point processes. Springer-Verlag, New York, 1993.

[7] Strassen, V. The existence of probability measures with given marginals, Ann. Math. Statist. 36 (1965), 423-439.

[8] Timár, Á. Tree and grid factors for general point processes, Elect. Comm. in Probab. 9 (2004), 53-59. 